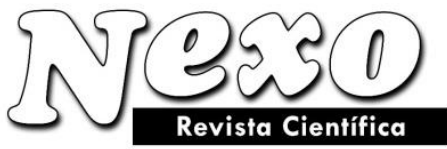

Universidad Nacional de Ingeniería COPYRIGHT @ (UNI). TODOS LOS DERECHOS RESERVADOS http://revistas.uni.edu.ni/index.php/Nexo https://doi.org/10.5377/nexo.v34i04.12635

\title{
Formulación de una espuma de remolacha (Beta vulgaris) a partir de lecitina de soya
}

\author{
Formulation of a beetroot foam \\ (Beta vulgaris) from soy lecithin
}

\author{
Jesús Escandell Comesaña ${ }^{1}$, Alexis Pascual Aguirre ${ }^{2}$, Héctor Zumbado Fernández ${ }^{3 *}$ \\ ${ }^{1}$ Instituto de Farmacia y Alimentos. Universidad de La Habana. Cuba \\ ${ }^{2}$ Grupo Hotelero Islazul. Sucursal Varadero. Matanzas. Cuba \\ ${ }^{3}$ Facultad de Ciencias de la Salud. Universidad Técnica de Manabí. Ecuador. \\ *hzumbadof@gmail.com
}

(recibido/received: 07-mayo-2021; aceptado/accepted: 18-agosto-2021)

\begin{abstract}
RESUMEN
El objetivo de esta investigación fue formular una espuma de remolacha estable en el tiempo, con adición de lecitina de soya. Se ensayaron 3 concentraciones del agente espumante $(0,5: 1,0$ y 1,5 $\% \mathrm{~m} / \mathrm{V}$ ) y se determinó la estabilidad mediante dos metodologías (suspensión y reposo). Se midió la viscosidad de las disoluciones en estudio y se evaluó el proceso de maduración de Ostwald en la espuma obtenida. La formulación de jugo de remolacha con 1,5\% m/V de lecitina de soya mostró mayor viscosidad y estabilidad de la espuma, mientras que el método más eficaz para mantener la estabilidad fue el reposo sobre el jugo de remolacha por un tiempo no mayor a 10 minutos. La espuma de remolacha fue evaluada sensorialmente por expertos con la categoría global de buena, destacándose el atractivo que aporta la misma en preparaciones tradicionales.
\end{abstract}

Palabras clave: estabilidad de espumas, lecitina de soya, remolacha.

\begin{abstract}
The purpose of this study is to formulate a beet foam stable over time with the addition of Soy lecithin. Three concentrations of the foaming agent were tested $(0.5,1.0$ and $1.5 \% \mathrm{w} / \mathrm{V})$ using two methodologies (Suspension and Standing) in order to determine the foam's stability. The viscocity of different dilutions were measured, and besides, the Ostwald maturation process was evaluated in the foam obtained. The beet juice formulation with $1.5 \% \mathrm{w} / \mathrm{V}$ soy lecithin had the highest viscosity, while the most effective procedure to maintain foam stability was the Standing method for no longer than 10 minutes. The beet foam was sensorially tested by experts giving the global category of good, highlighting the attractiveness it brings to traditional preparations.
\end{abstract}

Keywords: foam stability, soy lecithin, beet. 


\section{INTRODUCCIÓN}

En las últimas décadas la aplicación de la ciencia en las cocinas de chefs vanguardistas ha ido ganando terreno, ellos han encontrado en la "Gastronomía Molecular" un nuevo modelo de cocina ideal en el cual la química y la física tienen un papel protagónico.

El término "Gastronomía Molecular" fue implementado por el científico francés Hervé This y el físico húngaro Nicholas Kurti cuya aplicación no ha dejado de existir y para muchos su crecimiento ha sido desmedido. Esta tendencia gastronómica no implica únicamente el empleo de sustancias para lograr reacciones en los ingredientes; la gastronomía molecular representa también el estudio de los ingredientes naturales y las reacciones químicas que se producen en el alimento.

Las espumas son sistemas en los que un gas constituye la fase dispersa y un líquido o sólido la fase continua; la fase gaseosa se esparce a lo largo de toda la fase líquida, de manera que las cavidades o burbujas de gas quedan separadas por finas películas de líquido. La estabilidad de estas delgadas películas de líquido son las que determinan la estabilidad global de la espuma. Las dos fases que conforman la espuma están íntimamente ligadas y forman una estructura que se torna más ordenada a medida que la cantidad de gas aumenta (Santacruz, et al., 2015).

El agente espumante es un compuesto que se ubicará en la interfase gas-líquido provocando una disminución en la tensión superficial del líquido a espumar y la velocidad de formación de las burbujas debe ser mayor que la velocidad de colapso de las mismas, de manera que la espuma aumente su volumen a medida que transcurre el tiempo (Rodríguez, 2006; Valdivia, 2014).

A principios del siglo XXI el uso de emulsionantes con propiedades espumantes da lugar a lo que en cocina se han denominado "aires", que no son más que espumas, sistemas dispersos con un contenido de aire muy elevado, producidos con la ayuda de agitadores en la interfase gas-líquido (Mans y Castells, 2011).

La propuesta de esta investigación es una espuma de remolacha, la cual no aporta un sabor significativo, pero si un color llamativo y especial para el emplatado de disímiles preparaciones, pudiendo añadirse tanto en platos dulces como salados; realzando a la vista el color de la preparación y aumentando el interés del comensal por probar dicho plato. En Cuba no se han realizado investigaciones sobre formulación de espumas en el área de los alimentos, como tampoco se ha estudiado la estabilidad de las mismas.

En el Instituto de Farmacia y Alimentos de la Universidad de La Habana, existe una línea de investigación de Gastronomía Molecular y se han desarrollado estudios dirigidos a la evaluación del comportamiento de esferificaciones y su aplicación en diferentes productos (Pedroso, 2010; Borges, 2014; Barceló, 2016; Mandiarote, 2019; Escandell et al., 2021). Sin embargo la temática de las espumas aun no ha sido investigada.

Por todo lo anterior, se plantea el presente trabajo con el objetivo de formular una espuma de remolacha a partir de lecitina de soya con buena estabilidad.

\section{MATERIALES Y MÉTODOS}

La investigación se llevó a cabo en el Instituto de Farmacia y Alimentos de la Universidad de La Habana, en el Instituto de Investigaciones para la Industria Alimentaria y en el restaurante Atelier ubicado en calle 5ta, No. 511 altos e/ Paseo y 2, Vedado, La Habana. 


\subsection{Preparación del jugo de remolacha y obtención de la espuma}

Se utilizaron remolachas (Beta vulgaris) sanas y maduras adquiridas en un agromercado de La Habana. Se lavaron con agua y una disolución de 200 ppm de hipoclorito de sodio y se le realizaron cortes en la parte superior e inferior con un cuchillo desinfectado. Se procedió a quitar toda la piel de las mismas y se cortaron en rodajas. Se pesaron $2 \mathrm{~kg}$ de remolacha sin piel y en rebanadas, se les añadieron $500 \mathrm{~mL}$ de agua destilada y se trituraron en un homogeneizador (ULTRA - TURRAX, IKA T25 digital) durante 10 minutos a $24000 \mathrm{rpm}$. La pulpa obtenida se pasó a través de una tela de gasa estéril para la obtención del jugo.

Para la obtención de la espuma se adicionó a $100 \mathrm{~mL}$ del jugo de remolacha cantidades entre 0,5 y 1,5 g de lecitina de soya microencapsulada pesada en balanza analítica Sartorius, BSA 124S Max 120 g. La disolución se sometió a $20000 \mathrm{rpm}$ en un agitador ULTRA - TURRAX, IKA T25 digital, durante 5 minutos. Se ensayaron tres formulaciones con adición de 0,$5 ; 1,0$ y $1,5 \mathrm{~g}$ de lecitina de soya.

\subsection{Determinación de la viscosidad de las formulaciones}

Las propiedades de flujo de las formulaciones se realizaron en un Reómetro (Modular Compact Rheometer) MCR 302, Anton Paar, austriaco, a $25^{\circ} \mathrm{C}$ con la utilización de una copa cilindro de $27 \mathrm{~mm}$ (CC-27). La viscosidad media se determinó a la velocidad de $\mathrm{V} 50 \mathrm{~s}^{-1}$.

\subsection{Diseño de experimento}

Para evaluar la estabilidad de la espuma y seleccionar la mejor formulación, se realizó un diseño factorial $3 \times 5$ en el que se evaluó el efecto de los factores concentración de lecitina de soya con tres niveles $(0,5$, $1,0,1,5 \mathrm{~g} / 100 \mathrm{~mL})$ y tiempo de drenado con cinco niveles (2, 4, 6, 8 y $10 \mathrm{~min}$ ), así como la interacción entre ellos. La estabilidad se determinó mediante la medición del líquido de drenado gravitacional de la espuma en suspensión y del líquido de drenado de la espuma en reposo sobre el jugo. Se realizaron 3 réplicas para cada tiempo y disolución, para un total de 45 mediciones.

Determinación del drenado gravitacional del líquido en suspensión: se colocó la espuma dentro de un embudo de filtración de $60 \mathrm{~mm}$ de diámetro, cuyo vástago se introdujo en una probeta de $5 \mathrm{~mL}$ y se midió el volumen escurrido a diferentes tiempos (2, 4, 6, 8 y $10 \mathrm{~min})$. El volumen de espuma a introducir fue equivalente a la capacidad volumétrica del embudo y se estableció 10 minutos como tiempo máximo, pues las espumas son sistemas altamente inestables, y en los primeros minutos de su vida es donde se observa el mayor cambio de su estructura. El análisis se realizó por triplicado para cada tiempo con cada una de las concentraciones propuestas.

Determinación del drenaje del líquido de la espuma en reposo sobre el jugo de remolacha: en este método la espuma no estuvo suspendida, sino que reposó sobre el propio jugo de remolacha con lecitina de soya con el que fue elaborada. Una vez que se sometió la preparación a agitación y la espuma alcanzó el tope del vaso de precipitado se detuvo el proceso y se midió a partir de la línea divisoria entre el jugo remanente y la espuma sobrenadante el volumen drenado a diferentes tiempos (2, 4, 6, 8 y $10 \mathrm{~min})$. El análisis se realizó por triplicado para cada tiempo con cada una de las concentraciones propuestas.

\subsection{Análisis del comportamiento de las burbujas mediante el proceso de maduración de Ostwald}

Se tomaron instantáneas por microscopía óptica empleando un microscopio NOVEL, lente 10X, con cámara acoplada HDCE-50B, a diferentes tiempos (0, 2, 4, 6, 8 y 10 minutos) con el objetivo de examinar 
el comportamiento de las burbujas de la espuma de remolacha con la mejor formulación, mediante el proceso de la maduración de Ostwald.

2.5. Análisis descriptivo simple de la espuma de remolacha mediante expertos culinarios

Se realizó una evaluación sensorial de la espuma de remolacha a partir de lecitina de soya aplicada a diferentes preparaciones culinarias en el restaurante Atelier. La evaluación se llevó a cabo por expertos culinarios y especialistas en evaluación sensorial conocedores de la gastronomía molecular. Se utilizó el método de impresión general de la calidad descrito por Duarte (2013), con el cual los expertos describieron las características de apariencia, olor, sabor y textura y emitieron un dictamen global de la calidad sensorial del producto en una escala de cinco categorías desde 1 (Pésimo) a 5 (Excelente).

\subsection{Procesamiento de los resultados}

Los resultados de la medición de viscosidad y el diseño factorial $3 \times 5$ se procesaron por comparación múltiples de medias (ANOVA) y en los casos en que se encontraron diferencias significativas se aplicó la prueba de rangos múltiples de Duncan. Por otra parte, la comparación del volumen de drenado por ambos métodos utilizados para medir la estabilidad de las espumas, se realizó aplicando la prueba U de MannWhitney para contrastar dos muestras independientes. Todos los análisis se realizaron empleando el software estadístico IBM SPSS Statistics versión 22.

\section{RESULTADOS Y DISCUSIÓN}

\subsection{Determinación de la Viscosidad de las formulaciones}

Los resultados de la determinación de viscosidad evidencian diferencias significativas entre las tres disoluciones, correspondiendo a la formulación elaborada con 1,5\% de lecitina de soya los mayores valores de este parámetro (tabla 1)

Tabla 1. Viscosidad del jugo de remolacha a diferentes concentraciones de lecitina de soya

\begin{tabular}{cc}
\hline $\begin{array}{c}\text { Concentración de } \\
\text { lecitina de soya }(\%)\end{array}$ & Viscosidad (mPa.s) \\
\hline 0,5 & $1,82(0,02)^{\mathrm{c}}$ \\
1,0 & $3,05(0,02)^{\mathrm{b}}$ \\
1,5 & $6,02(0,04)^{\mathrm{a}}$ \\
\hline Letras diferentes denotan diferencias significativas $(\mathrm{p}<0,05)$ \\
Los valores se indican como media (desviación estándar)
\end{tabular}

Del conjunto de factores que afectan el caudal de drenaje, la viscosidad es el más relevante. A medida que este aumenta, debe incrementarse la estabilidad de la espuma, ya que el caudal de drenaje disminuye (Mangas y Blanco, 2010). Algunos autores mencionan que la mejor cohesión entre burbujas y la mejor estabilidad de las capas que recubren el aire, resulta en una mayor viscosidad y produce una transferencia gaseosa más difícil a través de las películas, por lo que este efecto debe mejorar la estabilidad de manera apreciable (Salager et al., 1999). Estos resultados permiten inferir la posibilidad de que la formulación con $1,5 \%$ de lecitina exhiba una mayor estabilidad. 


\subsection{Determinación del drenaje de líquido de la espuma en suspensión}

En la tabla 2 se muestran los resultados del volumen drenado de la espuma en suspensión con diferentes concentraciones de lecitina de soya y a diferentes tiempos.

Tabla 2. Volumen drenado de la espuma en suspensión

\begin{tabular}{cccc}
\hline \multirow{2}{*}{ Tiempo $(\min )$} & \multicolumn{3}{c}{ Concentración de lecitina (\%) } \\
\cline { 2 - 4 } & $\mathbf{0 , 5}$ & $\mathbf{1 , 0}$ & $\mathbf{1 , 5}$ \\
\hline 2 & $1,33(0,58) \mathrm{a}$ & $1,17(0,06) \mathrm{a}$ & $1,17(0,06) \mathrm{a}$ \\
4 & $1,50(0,10) \mathrm{a}$ & $1,40(0,20) \mathrm{a}$ & $1,23(0,15) \mathrm{a}$ \\
6 & $1,70(0,26) \mathrm{a}$ & $1,77(0,15) \mathrm{b}$ & $1,43(0,06) \mathrm{b}$ \\
8 & $1,97(0,35) \mathrm{ab}$ & $2,00(0,26) \mathrm{bc}$ & $1,57(0,06) \mathrm{b}$ \\
10 & $2,23(0,25) \mathrm{b}$ & $2,13(0,15) \mathrm{c}$ & $1,63(0,15) \mathrm{b}$ \\
Total & $1,75^{1}$ & $1,69^{1}$ & $1,41^{2}$ \\
\hline
\end{tabular}

Letras diferentes en las columnas denotan diferencias significativas $(\mathrm{p}<0,05)$

Números diferentes en la última fila denotan diferencias significativas $(\mathrm{p}<0,05)$

Los valores se indican en $\mathrm{mL}$ como media (desviación estándar)

Nótese que a medida que transcurre el tiempo aumentan los volúmenes drenados, pero en los primeros minutos, para las tres concentraciones, se observa que no hay diferencias significativas entre los volúmenes drenados. Según el estudio de Ruiz (2016), al utilizar espumas estabilizadas con un tensoactivo drenando con geometría de embudo, el número de bordes de Plateau disminuye conforme se desplazó en la espuma, lo que significa que, para tiempos cortos, en una región fija de la espuma, el volumen de líquido que entra es menor al volumen de líquido que sale, lo que provoca que, a tiempos cortos, el valor de volumen drenado sea aparentemente uniforme.

Entre las concentraciones $0,5 \%$ y $1,0 \%$ no existen diferencias significativas entre los volúmenes de drenado, pero ambos difieren de 1,5\%, la cual presentó el menor escurrimiento en el tiempo. Al aumentar la concentración de lecitina aumenta la viscosidad del jugo de remolacha (tabla 1), lo que provoca que la espuma sea mucho más estable y el volumen drenado sea menor (Mangas y Blanco, 2010; Galindo et al., 2014). Se selecciona entonces la concentración de $1,5 \% \mathrm{~m} / \mathrm{V}$ como la mejor para formular una espuma más estable en el tiempo.

\subsection{Determinación del drenaje del líquido de la espuma en reposo sobre el jugo de remolacha}

En este caso la espuma no estuvo suspendida en un embudo, sino que reposó sobre el jugo de remolacha con lecitina de soya con el que fue creada. En la tabla 3 se observa un comportamiento análogo al obtenido con el método de drenado en suspensión en relación con el parámetro tiempo; sin embargo, el volumen total drenado no presenta diferencias significativas entre las diferentes concentraciones. No obstante, se selecciona como mejor formulación la concentración de 1,5\% porque pequeños cambios en el volumen drenado pueden dar lugar a grandes cambios en la estructura de la espuma, dado que las capas de líquido son las encargadas de encapsular el aire en su estructura. La mayor viscosidad de la formulación al $1,5 \%$ es otro elemento que determinó su selección. 
Tabla 3. Volumen drenado de la espuma en reposo sobre el líquido

\begin{tabular}{cccc}
\hline \multirow{2}{*}{ Tiempo $(\min )$} & \multicolumn{3}{c}{ Concentración de lecitina (\%) } \\
\cline { 2 - 4 } & $\mathbf{0 , 5}$ & $\mathbf{1 , 0}$ & $\mathbf{1 , 5}$ \\
2 & $1,00(0,10)^{\mathrm{a}}$ & $0,97(0,06)^{\mathrm{a}}$ & $0,93(0,15)^{\mathrm{a}}$ \\
4 & $1,13(0,06)^{\mathrm{a}}$ & $1,13(0,06)^{\mathrm{b}}$ & $1,13(0,15)^{\mathrm{ab}}$ \\
6 & $1,37(0,06)^{\mathrm{b}}$ & $1,30(0,10)^{\mathrm{bc}}$ & $1,20(0,10)^{\mathrm{ab}}$ \\
8 & $1,53(0,12)^{\mathrm{c}}$ & $1,30(0,10)^{\mathrm{bc}}$ & $1,27(0,21)^{\mathrm{b}}$ \\
10 & $1,57(0,06)^{\mathrm{c}}$ & $1,47(0,12)^{\mathrm{c}}$ & $1,40(0,10)^{\mathrm{b}}$ \\
Total & $1,32{ }^{\mathrm{c}}$ & $1,23^{1}$ & $1,19^{1}$ \\
\hline
\end{tabular}

Letras diferentes en las columnas denotan diferencias significativas $(\mathrm{p}<0,05)$

Números iguales en la última fila indican no diferencias significativas ( $p>0,05)$

Los valores se indican en $\mathrm{mL}$ como media (desviación estándar).

Al comparar los resultados de ambos métodos de drenado mediante la prueba $U$ de Mann-Whitney para muestras independientes se encontraron diferencias significativas (figura 1).

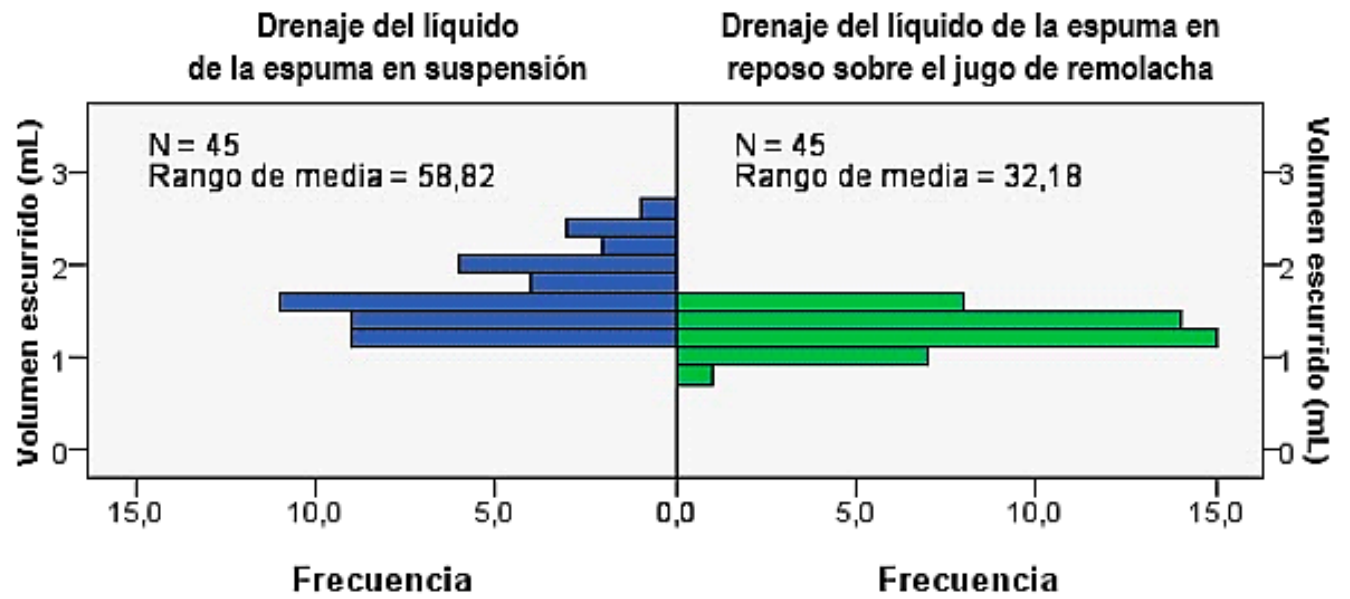

Figura 1. Prueba U de Mann-Whitney para muestras independientes.

El drenaje de la espuma sobre el líquido presenta menor volumen escurrido con respecto al drenaje de la espuma en suspensión sobre un embudo, lo que significa que la espuma que reposa sobre el líquido con el que fue formada, drena menos que si se mantiene en suspensión. Este resultado se puede explicar por el efecto de capilaridad de los líquidos, mediante el cual, el jugo de remolacha asciende por las películas de líquido entre las burbujas de aire en contra de la gravedad (Sancho, 2007). Al subir el líquido hacia la espuma, esta refuerza las películas que atrapan al aire y las hace más resistentes y, por tanto, más estables en el tiempo.

Los resultados obtenidos permiten recomendar al personal gastronómico que desee emplear esta espuma para decorar platos o cócteles, que dejen reposar la espuma formada en dicho jugo y no la coloquen en otro recipiente para que mantenga su estabilidad y por tanto su estructura. 


\subsection{Análisis del comportamiento de las burbujas mediante el proceso de maduración de Ostwald}

La figura 2 muestra la evolución en el tiempo de la espuma con 1,5 g de lecitina de soya en $100 \mathrm{~mL}$ de jugo de remolacha

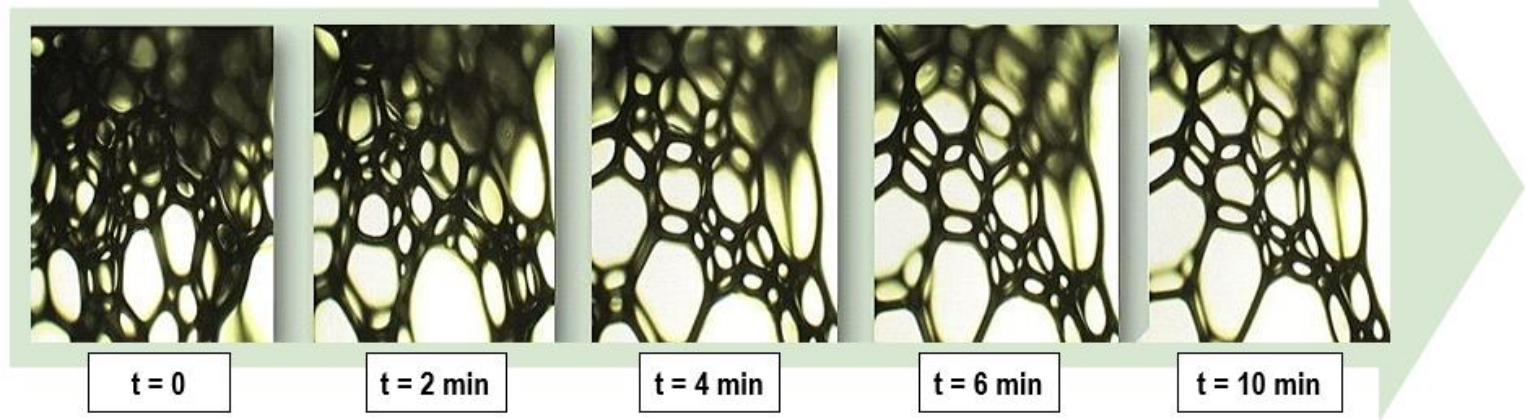

Figura 2. Evolución de la estructura de la espuma de remolacha en el tiempo.

Del análisis de esta figura se denota que a medida que aumenta el tiempo, disminuye el número de burbujas y éstas extienden considerablemente su tamaño. Una vez que se alcanzan los 6 minutos la espuma estabiliza su estructura sin observarse cambios perceptibles a simple vista.

El fenómeno de maduración de Ostwald, conduce a que las pequeñas burbujas se desinflen lentamente en las más grandes hasta desaparecer. En otros términos, el número de burbujas disminuyen y devienen grandes poco a poco sin que sea necesario que las películas se rompan (Salager et al., 1999).

Los resultados obtenidos demuestran que el proceso de maduración de Ostwald se presenta fuertemente marcado, y que a un valor superior a 10 minutos la espuma perderá rigidez y se debilitará su estructura, dejando escapar el aire contenido en sus películas de líquido. Se sugiere entonces que el tiempo entre la preparación de la espuma y el servicio gastronómico no debe exceder los 10 minutos.

\subsection{Análisis descriptivo simple de la espuma de remolacha mediante expertos culinarios}

La evaluación sensorial se realizó integrando la espuma en preparaciones culinarias del restaurante Atelier. Se seleccionó un entrante (Tapas de Vegetales) y dos platos fuertes (Sushi, y Ropa Vieja) (Figura 3).

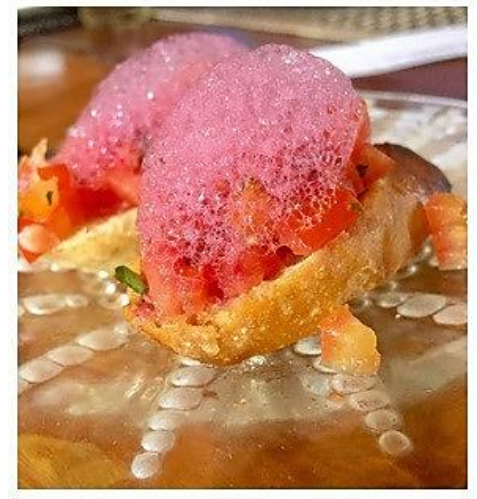

(a)

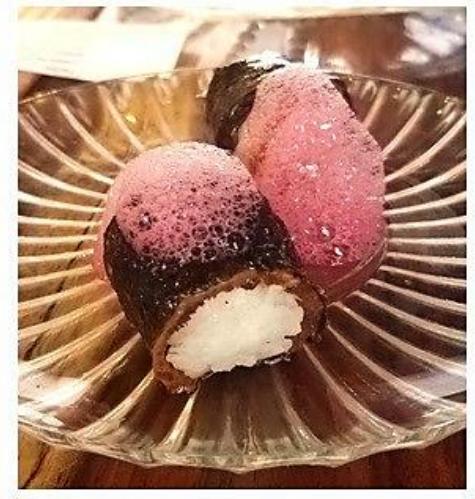

(b)

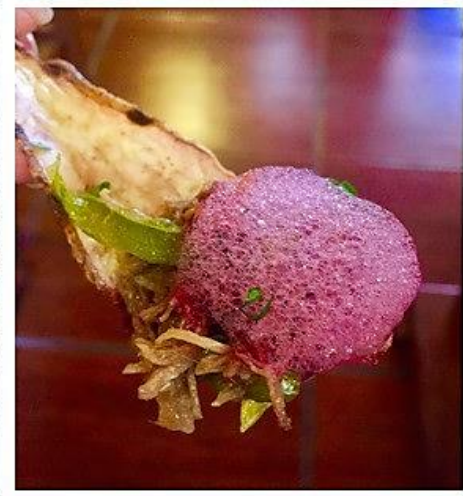

(c)

Figura 3. Platos con la espuma de remolacha como decoración. (a) Tapas de Vegetales, (b) Sushi, (c) Ropa Vieja. 
En esta prueba participaron 6 expertos culinarios, de los cuales, tres calificaron de excelente la calidad del producto, uno la señaló como buena y dos dictaminaron la misma como aceptable. Sobre su calidad sensorial global, se dictaminó que la espuma presenta buena apariencia, el olor es poco perceptible y su sabor es poco marcado. Plantearon además que la estructura de la espuma es bien definida y resaltó la imagen de las preparaciones dándole un nuevo atractivo que incita al cliente a consumir.

\section{CONCLUSIONES}

La formulación de jugo de remolacha con $1,5 \% \mathrm{~m} / \mathrm{V}$ de lecitina de soya resultó ser la de mayor viscosidad y estabilidad de la espuma en el tiempo.

El método más eficaz para mantener la estabilidad de las espumas es conservándolas en reposo sobre el líquido, al escurrir significativamente menos que en suspensión.

Al analizar el proceso de maduración de Ostwald en la espuma, se sugiere que el tiempo entre la preparación de la espuma y el servicio gastronómico no debe exceder los 10 minutos.

La espuma de remolacha fue evaluada sensorialmente con categoría global de 'Buena' destacándose el atractivo que aporta la misma a preparaciones tradicionales sin afectar su sabor original.

\section{REFERENCIAS}

Barceló, F. (2016). Influencia de la esferificación inversa en las propiedades organolépticas del coctel mojito. Tesis en opción al título en Licenciatura en Ciencias Alimentarias, Universidad de La Habana, La Habana, Cuba.

Borges, L. (2014). Influencia del grado alcohólico y el contenido de azúcar en la esferificación directa de licores. Tesis en opción al título en Licenciatura en Ciencias Alimentarias, Universidad de La Habana, La Habana, Cuba.

Duarte, C. (2013). Métodos objetivos para el control de la calidad sensorial. Ciencia y Tecnología de los Alimentos, 2(23), 12-17.

Escandell, J., Molina, I., \& Zumbado, H. (2021). Efecto de saborizantes en la esferificación inversa de un modelo de coctel. Ciencia y Tecnología de Alimentos, 31(1).

Galindo, E., Corkidi, G., Holguín, A., \& López, D. (2014). Producción de espuma en el chocolate con el molinillo tradicional. Revista Digital Universitaria, 15(5), 2-11.

Mandiarote, A. (2019). Efecto de los colorantes Sunset Yellow y Rojo Ponceau 4R en la esferificación inversa de un modelo de cóctel. Tesis en opción al título en Licenciatura en Ciencias Alimentarias, Universidad de La Habana, La Habana, Cuba.

Mangas, J., \& Blanco, D. (2010). La manzana y la sidra: bioprocesos, tecnologías de elaboración y control. (D. Blanco Gomez, \& J. Mangas Alonso, Edits.) Asturias, España: Serida.

Mans, C., \& Castells, P. (2011). La nueva cocina científica. Investigación y Ciencia(421), 56-63. 
Pedroso, D. (2010). Influencia de la esferificación directa en las propiedades sensoriales de una salsa criolla. Tesis en opción al título en Licenciatura en Ciencias Alimentarias, Universidad de La Habana, La Habana, Cuba.

Rodríguez, L. (2006). Sistemas Coloidales en Farmacia. Emulsiones y espumas. Guanajuato: Salamanca.

Ruiz, M. (2016). Procesos Biofarmacéuticos: Su relación con el diseño de formas farmacéuticas y el éxito de la farmacoterapia. La Plata: Universidad Nacional de La Plata. Facultad de Ciencias Naturales y Museo.

Salager, J., Andérez, J., \& Firgiarini, A. (1999). Influencia de la formulación sobre las espumas. L'actualite chimique, 10-21.

Sancho, J. (2007). Agua es vida. Revista de la Academia de Ciencias Exactas, Físicas, Químicas y Naturales de Zaragoza(62), 65-74.

Santacruz, V., Santacruz, C., \& Laguna, J. (2015). Physical characterization of freeze-dried foam prepared from aloe vera gel and guar gum. Vitae, Revista de la facultad de ciencias farmacéuticas y alimentarias, $22,75-86$.

Valdivia, M. (2014). Espumas en alimentos. Revista Digital Universitaria, 15(5), 2-9.

\section{SEMBLANZA DE LOS AUTORES}
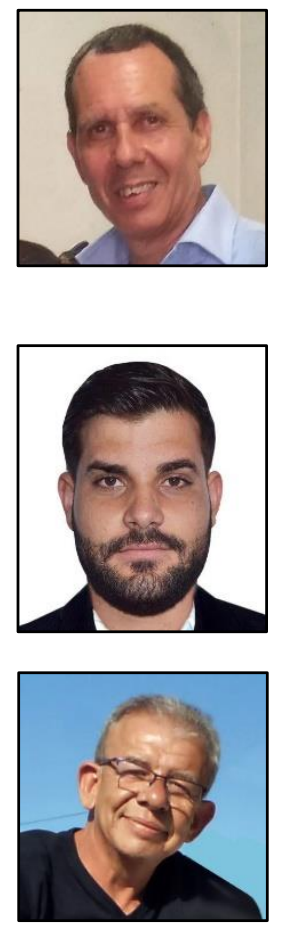

Jesús V. Escandell Comesaña: Cubano. Master en Didáctica de la Química (2002). Licenciado en Química (1983). Profesor del Instituto de Farmacia y Alimentos de la Universidad de La Habana. Posee amplia experiencia docente en pregrado y posgrado en la impartición de las asignaturas Química Física y Bioestadística. Autor de artículos científicos y del libro de texto Cinética Química para Farmacia y Alimentos. Jefe de la Línea de Investigación de Gastronomía Molecular.

Alexis M. Pascual Aguirre: Cubano. Licenciado en Alimentos (2019). Adiestrado en Gerencia A+B (Alimentos y Bebidas) en el Departamento de Operaciones del Grupo Hotelero Islazul, Sucursal Varadero. Colaborador del Proyecto de Investigación de Gastronomía Molecular.

Héctor M. Zumbado Fernández: Cubano. Doctor en Ciencias Pedagógicas (2005). Master en Ciencia y Tecnología de los Alimentos (1998). Licenciado en Alimentos (1985). Ha impartido docencia en pregrado y postgrado en temáticas de Análisis de los Alimentos, Química de los Alimentos, Metodología de la Investigación Científica, Didáctica Universitaria y Comunicación Educativa, entre otras. Autor de 5 libros de texto y más de 40 publicaciones científicas. Docente de la Universidad Técnica de Manabí y colaborador del Proyecto de Investigación de Gastronomía Molecular. 